\title{
The Molecular Mechanism of Organelle Transport along Microtubules: The Identification and Characterization of Kifs (Kinesin Superfamily Proteins)
}

\author{
Nobutaka Hirokawa \\ Department of Anatomy and Cell Biology, Faculty of Medicine University of Tokyo, 7-3-1 Hongo, Tokyo 113, \\ Japan
}

\begin{abstract}
$A B S T R A C T$. In the cells various kinds of organelles are transported and distributed to their proper destinations in the cell. Organelle transports are very important for cellular morphogenesis and functions, with the conveying and targeting of essential materials to their correct destination being conducted, often at considerable velocities. Recently we have identified at least 10 new microtubule-associated motor proteins named as KIFs (kinesin superfamily proteins). Their characterization reveals that each member can convey a specific organelle or cargo, although there is some redundancy. It has also become clear that there are distinct subclasses of KIFs that form monomeric, heterodimeric and homodimeric motors. Molecular cell biological approaches combining multidiciplinary methods such as new electron microscopy, biochemistry, immunocytochemistry, biophysics, molecular biology and molecular genetics have revealed precise mechanisms of organelle transports in the cells by KIFs.
\end{abstract}

Cells need to distribute various distinct kinds of proteins to their proper destination after synthesis. Many proteins are transported as integral membrane proteins or as proteins associated with membranes. This transport is very important for the proper targeting of proteins to distinct parts of cells, especially in highly polarized and differentiated cells such as epithelial cells and neurons. The neuron is a typical polarized cell composed of branching dendrites, cell body and a long axon along which impulses are propagated. Because of the lack of protein synthesis machinery in the axon (the axon can be up to $1 \mathrm{~m}$ long in human) most of the materials needed in the axon and synapses have to be transported down the axon after the synthesis in the cell body (1). In this sense, axonal transport is fundamental for neuronal cell morphogenesis and function, and consequently the neuronal axon provides an excellent model system in which to study organelle transport in cells in general (1). Two types of axonal transport occur in neurons, fast and slow. Fast transport is responsible primarily for the movement of membranous organelles at 50-200 mm day ${ }^{-1}$ towards the synapse (anterograde transport) or back to the cell body (retrograde transport). By contrast, slow transport moves cytoskeletal proteins and 'soluble' enzymes at $0.5-4 \mathrm{~mm}$ day $^{-1}$. The recent discovery and characterization of kinesin superfamily proteins (KIFs) sheds new light on the mechanisms of membranous organelle transport. In this article I review the molecular mechanisms of the organelle transport based on data derived mainly from our laboratory.

\section{Molecular Mechanism of Organelle Transports}

I) The Molecular Structure and Role of Kinesin in vivo.

Various kinds of membranous organelles are transported in nerve axons, where transport is bidirectional and occurs at varying velocities. Membranous organelles that move in an anterograde direction include mitochondria and tubulovesicular structures that could be precursors of axonal plasma membranes, synaptic vesicles and synaptic plasma membranes. These membranous organelles are likely to contain neuropeptides, neurotransmitters and associated enzymes, as well as some membrane-associated proteins. In contrast, membranous organelles moving in the retrograde direction include prelysosomal membranous organelles, multivesicular bodies, multilamellar bodies, mitochondria and endosomes (1). Neurotrophic factor receptors are also transported by this retrograde direction.

Early electron microscopic studies identified short (25-30 nm long) crossbridges between membranous organelles and microtubules, prompting the idea that these might be motor proteins responsible for fast transport $(2,7,8)$. The application of VE-DIC (video-enhanced differential interferance contrast) microscopy has facilitated the elucidation of the mechanisms of membrane organelle transport $(3,4)$. This approach enabled the clear visualization in real time of the bidirectional movements of several different kinds of membranous organelles along the axon and proved that the membranous organelles move along microtubules. Such studies led to the discovery of two microtubule-acti- 
vated ATPases, kinesin and brain (cytoplasmic) dynein (3-5). Kinesin transports beads from the minus end to the plus end of microtubules (anterograde) at $\sim 0.6 \mu \mathrm{m}$ $\mathrm{sec}^{-1}$, whereas brain dynein acts as a retrograde transporter.

Kinesin is a tetramer composed of two heavy chains and two light chains (6). These form a $80 \mathrm{~nm}$ rod-like molecule consisting of two globular head domains, a stalk domain and a fan-like tail domain. Decoration with antibodies specific for the heavy and light chains demonstrated that the heavy chains form heads, whereas the light chains form the fan-like ends (9). The heads bind to microtubules, and the fan-like ends are probably associated with membranous organelle (9). This molecular structure fits very well with the secondary structure predicted from the primary structure of Drosophila kinesin (10). Molecular genetic approaches have revealed that kinesin heads are responsible for the movement of kinesin on the microtubules.

Afterwards, several new approaches led to an expansion in our understanding of motor proteins and organelle transport. Analysis of high-resolution three-dimensional structures of kinesin heads and microtubules by the cryoelectron microscopy of frozen hydrated materials showed that kinesin heads bind quite regularly on ridges of microtubule protofilaments $(11,12)$ (Fig. 5). This fits with the observation that kinesin moves in a straight line on microtubules in in vitro motility assays (36). Furthermore, the kinesin heads bind asymmetrically to the microtubules depending on the polarity (12) (Fig. 5). Further studies on the dynamic conformational changes in the complex between kinesin heads and microtubules during hydrolysis of ATP should certainly be informative.

Although in vitro motility assays suggested kinesin is an anterograde motor in the cells, the evidence in vivo were lacking. By immunocytochemistry of ligated axons in vivo it became possible to show that kinesin is primarily associated with anterogradely moving membranous organelles which accumulate at the proximal end of ligation suggesting strongly that kinesin works as a motor for anterograde transport of these organelles (13).

II) Identification and Functions of New Microtubulebased Motor Proteins, KIFs (Kinesin superfamily proteins).

Various kinds of membranous organelles are conveyed bidirectionally in nerve axons at different velocities. Electron microscopic observations have suggested that the detailed structures of crossbridges between mi- crotubules and different kinds of membrane organelles are distinct $(2,7,8,33)$ (Fig. 1). On these bases we thought that it seems unlikely that only two unidirectional motor proteins, namely, kinesin and brain dynein, could be responsible for precisely transporting all the various organelles correctly to their target points at correct velocities. Furthermore, the motors for slow transport of cytoskeletal proteins are still quite unknown. Therefore, we decided to search for new microtubule-dependent motor proteins (15).

In order to search systematically for novel, putative motors for organelle transport, we cloned cDNAs encoding both the ATP binding and microtubule binding consensus sequences from KHC and characterised from murine brain cDNA libraries (16). The cDNAs encode a superfamily of KIF proteins. Initially, five members, KIF1 (now known as KIF1A (17)), KIF2, KIF3 (now called KIF3A (18)), KIF4 and KIF5 were identified, but further members have now been described (KIF1B (19); KIF3B (20); KIFC1, KIFC2 and KIFC3; unpublished data from Kondo et al., Satio et al., and Noda et al.) (Fig. 2). These ten new putative motors can be categorized into three types: $\mathrm{N}$-terminal motor domain type such as KIF1A, KIF1B, KIF3A, KIF3B, KIF4 and KIF5; central motor domain type such as KIF2; and Cterminal motor domain types such as KIFC1, KIFC2 and KIFC3 (33). All these putative motors have been characterized by molecular cell-biological approaches, including cloning and sequencing, expression and purification using the baculovirus Sf9 cell system, observation of molecular structures by EM, in vitro motility assay, immunocytochemistry and identification of cargoes by subcellular fractionation and immunoprecipitation (33).

KIF1A (17) is a novel, monomeric, neuron-specific KIF and has the fastest reported anterograde motor activity $\left(1.5 \mu \mathrm{m} \mathrm{sec}^{-1}\right)$. The membranous organelles carried by KIF1A contain some synaptic vesicle proteins, such as synaptotagmin, synaptophysin and Rab3A, but not others such as SV2, presynaptic membrane proteins, in particular, syntaxin 1A or SNAP-25, or other known anterograde motors, such as kinesin, KIF3A and KIF3B. Thus, KIF1A is a unique monomeric anterograde motor for transport of a subtype precursor of synaptic vesicle containing synaptotagmin, synaptophysin and Rab3A. This work clearly indicated also that synaptic vesicles are transported as at least two precursors and could be derived through exocytosis and endocytosis cycle in the terminals (Figs. 2, 3).

KIF1B (19) is an N-terminal type motor protein ex-

\footnotetext{
Fig. 1. Quick frozen, deep-etched axons in which various kinds of crossbridges (arrows) are identified between membranous organelles and microtubules. (a). A crossbridge (arrow) composed of two heads and a longer stalk ( $\sim 25 \mathrm{~nm}$ ). (b). Only globular heads (arrows) are recognizable between membranous organelle and a microtubule. (c). Crossbridges (arrows) between mitochondria and a microtubule are short and have small heads. Bar, $50 \mathrm{~nm}$.
} 

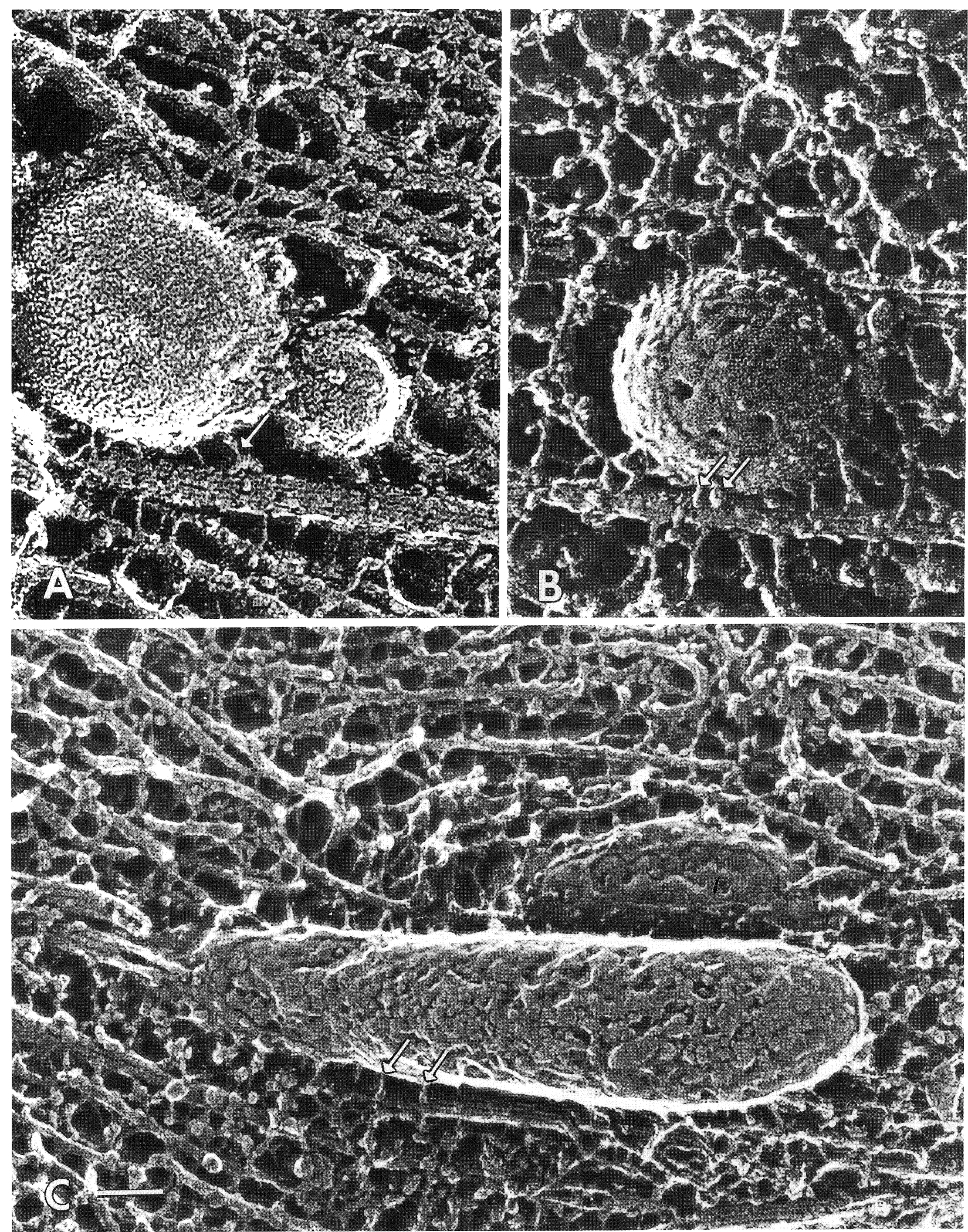

Fig. 1. 
pressed ubiquitously in various kinds of tissues including neurons. KIF1B is also a unique monomeric globular motor, occasionally showing a short tail in electron micrographs and possessing a plus end-directed motility on microtubules $\left(0.6 \mu \mathrm{m} \mathrm{sec}^{-1}\right)$. Immunocytochemistry and subcellular fractionation show the association of KIF1B with mitochondria. Furthermore, purified KIF1B can transport mitochondria along microtubules

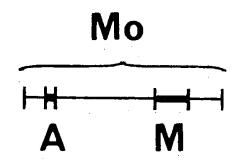

KIF1A

KIF1B

KIF2

KIF3A

1

KIF3B

KIF4

KIF5

(bKHC)

uKHC
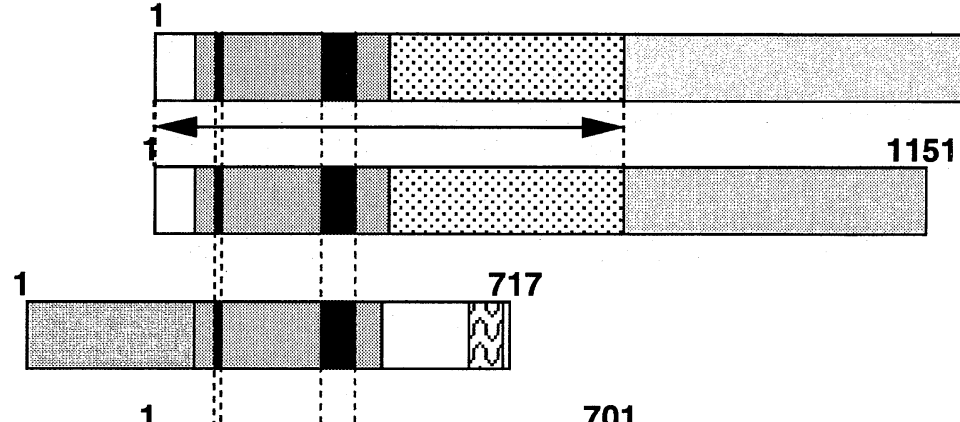

701

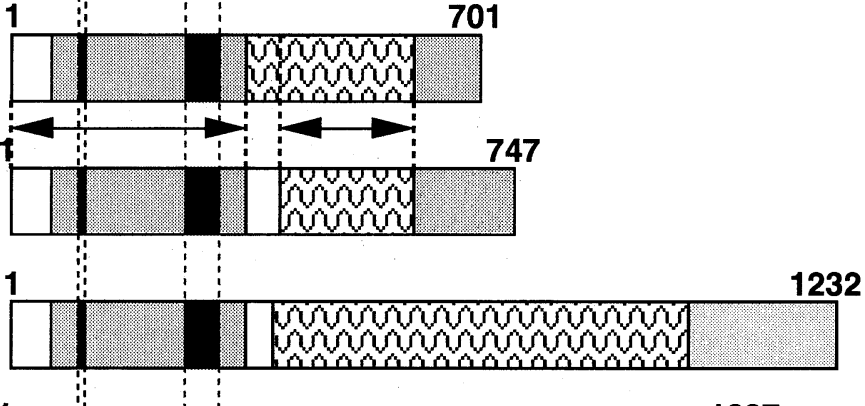

KIFC1

KIFC2

KIFC3

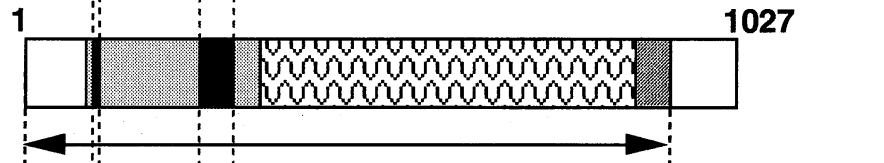

961

Fig. 2. Structure of cDNAs for murine kinesin superfamily proteins (KIFs). Mo, motor domain; A, ATP-binding consensus sequence; M, microtubule-binding consensus sequence. KIF1A, KIF1B, KIF3A, KIF3B, KIF4, KIF5 [brain kinesin (bKHC)] and uKHC (ubiquitous kinesin) are $\mathrm{N}$-terminal motors, whereas KIF2 is a central-motor-domain type. KIFC1, KIFC2 and KIFC3 are C-terminal-motor-domain types. Arrows between pairs of motor proteins indicate regions sharing strong homology. 
in vitro (Fig. 4). Thus, there is a strong suggestion that KIF1B works as a monomeric motor for anterograde transport of mitochondria.

KIF2 (21) is a member of the central-motor-domain type and belongs to a new family. KIF2 is expressed in juvenile neurons and expression is reduced in the adult. Expressed predominantly in neurons and to a lesser degree in other tissues, it forms a dimer showing microtubule plus-end-directed motor activity $\left(\sim 0.5 \mu \mathrm{m} \mathrm{sec}^{-1}\right)$. It works as an anterograde motor in juvenile axons and conveys a particular group of membranous organelles (100-120 nm in diameter) that are distinct both from synaptic vesicle precursors and from vesicles conveyed by other motors, such as kinesin, KIF3A and KIF3B. Thus, KIF2 is an anterograde motor whose expression is regulated developmentally (Figs. 2, 3).

KIF3A (18) is an N-terminal type motor and was the first representative of a new KIF3 family. Subsequently, KIF3B (20) was identified, whose motor domain is partially homologous $(69 \%)$ to the KIF3A motor domain. Interestingly, although KIF3A and KIF3B are derived from different genes, they form a heterodimer and work as a plus-end-directed motor on microtubules $(0.3 \mu \mathrm{m}$ $\mathrm{sec}^{-1}$ ). They are expressed strongly in neurons and testis and less abundantly in other tissues. The KIF3AKIF3B heterodimer forms a $50 \mathrm{~nm}$ rod with two globular heads (diam. $\sim 10 \mathrm{~nm}$ ) at one end and a small globu- lar structure at the other end. This KIF3A-KIF3B heterodimer transports vesicles (dia. $90-160 \mathrm{~nm}$ ) that are distinct from synaptic vesicle precursors. Interestingly, the native KIF3 complex $(\sim 350 \mathrm{kDa})$ in brain is composed of KIF3A-KIF3B heterodimer and other high molecular weight $(\sim 100 \mathrm{kDa})$ associated polypeptides called the kinesin-superfamily-associated protein 3 (KAP3) [KAP3A (793 aa) and KAP3B (772 aa)] $(20,34)$. In vitro reconstitution study proved that KAP3 is a globular protein that binds to the tail end of KIF3A-KIF3B heterodimer, forming $\mathrm{KIF} 3 \mathrm{~A} / 3 \mathrm{~B} \cdot \mathrm{KAP} 3$ heterotrimer. Thus, KIF3A-KIF3B seems to work as a novel heterodimeric motor with KAP3 to transport vesicles in the anterograde direction (20) (Figs. 2, 3). Because KAP3 did not affect ATPase activity of KIF3A/3B heterodimer and because KAP3 is associated with the tail domain of $\mathrm{KIF} 3 \mathrm{~A} / 3 \mathrm{~B}$, where it is assumed to be involved in the binding of KIF3A/3B to cargoes, KAP3 may regulate the binding of KIF3A-KIF3B to its cargoes (34).

KIF4 (22) is an N-terminal-motor-domain type, homodimeric motor forming a 116-nm rod with two globular heads and a tail, and belongs to yet another new family, the KIF4 family. It is a plus-end-directed motor on microtubules $\left(\sim 0.2 \mu \mathrm{m} \mathrm{sec}^{-1}\right)$ and is expressed strongly in juvenile tissues, including young but differentiated neurons, whereas its expression decreases considerably in adult mice, except in spleen. KIF4 colocalizes with

\section{KIF1A}

KIF1B

KIF2

KIF3A/B

KIF4

KHC

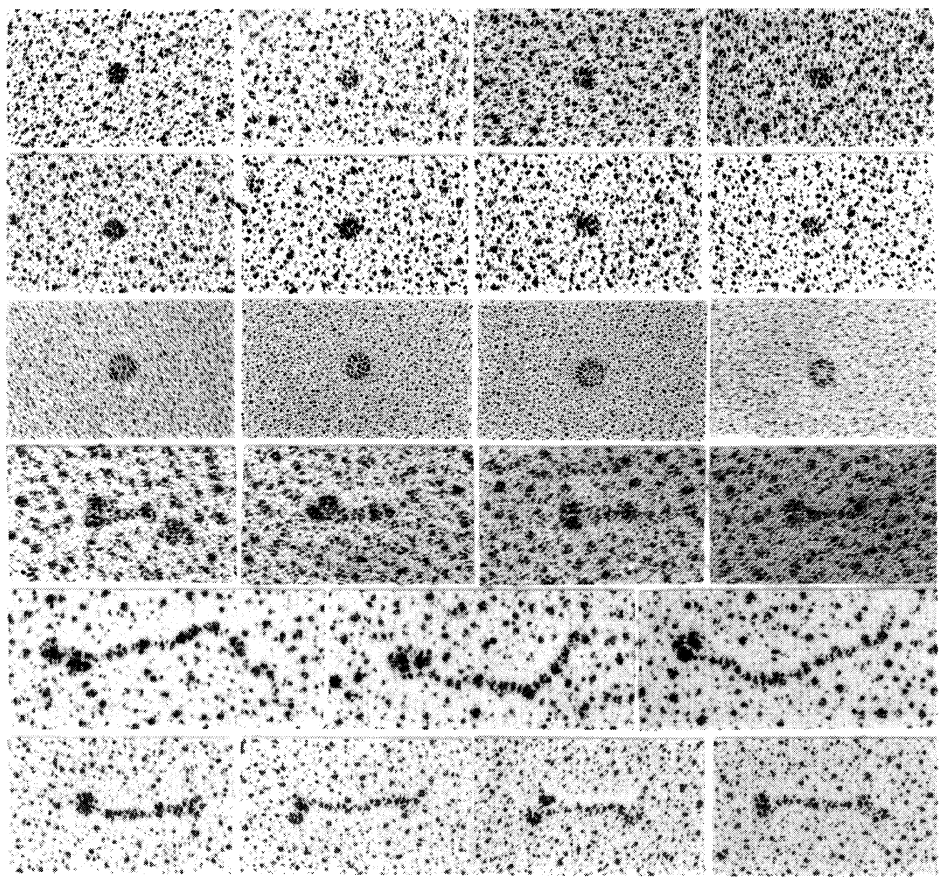

Fig. 3. Structures of KIFs observed by the low angle rotary shadowing EM. KIF1A; monomer (Ref. 17), KIF1B; Monomer (Ref. 19), KIF2; homodimer (Ref. 21), KIF3A/3B; heterodimer (Ref. 20), KHC; homodimer (Ref. 9). Bar, $100 \mathrm{~nm}$. 

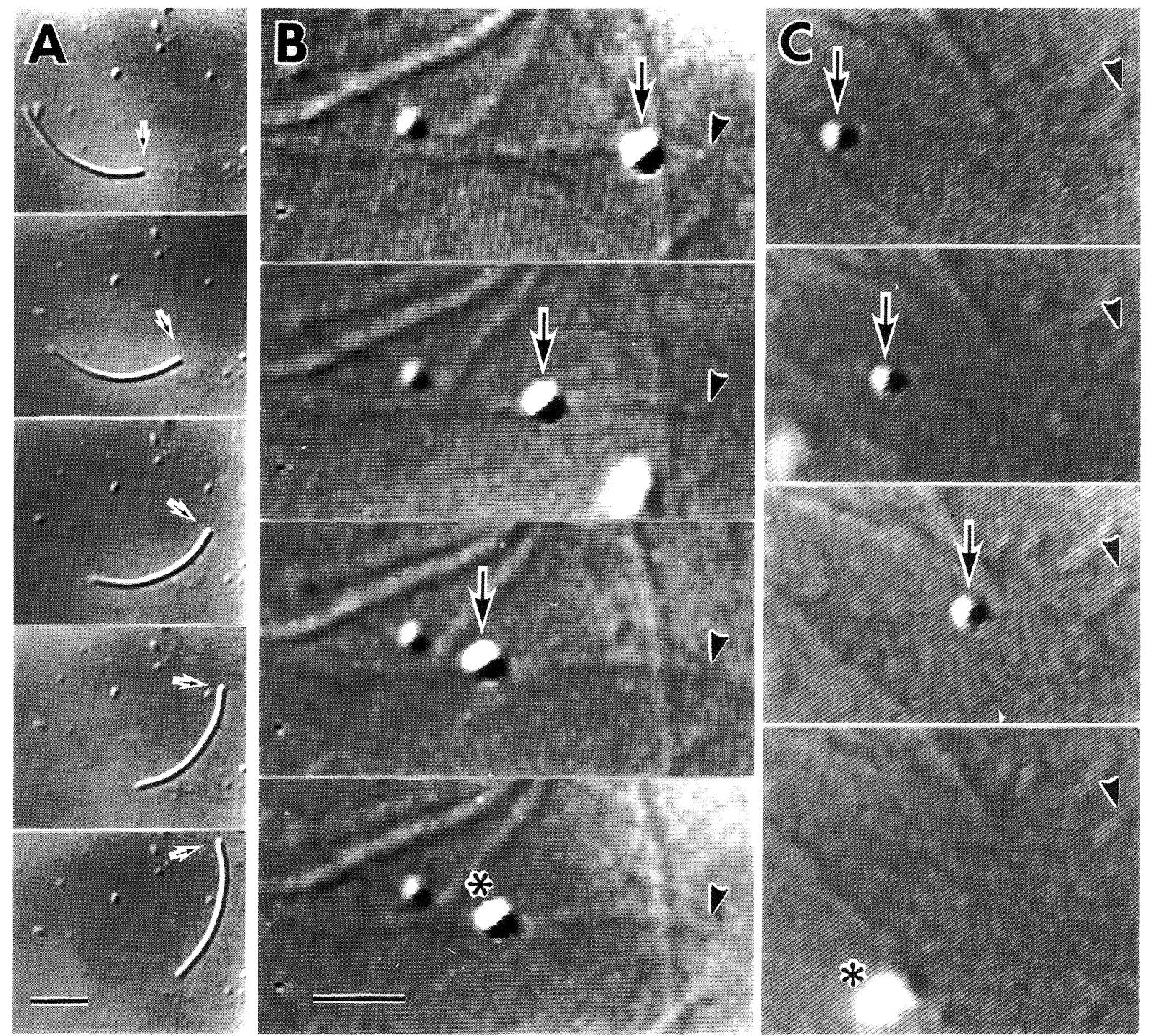

Fig. 4. In vitro motility assay of KIF1B. The movement of axonemes on recombinat KIF1B-coated coverslips was observed in (A). The pictures were taken at 30 s intervals. Arrows indicate the tips of moving axonemes. The axonemes glided toward their compact end, indicating plus end-directed motility activity of the KIF1B protein. Bar, $5 \mu \mathrm{m}$. In (B) and (C) two series of photographs show that mitochondria incubated with KIF1B moved unidirectionally along microtubules. In (B), taken at $30 \mathrm{sec}$ intervals, the mitochondria (arrow) moved rapidly along a microtubule. The railway microtubule, the ends of which are indicated by big arrowheads, is indicated by small arrowheads (Ref. 19).

membranous organelles both in the growth cones of differentiated neurons and in the cytoplasm of cultured fibroblasts. Thus, KIF4 is a novel anterograde motor whose expression is regulated developmentally.
On the bases of these molecular call biological studies of KIFs it is now clear that there are many distinct KIFs in addition to kinesin that could be used for anterograde transports. The discovery and characterization of

Fig. 5. Three-dimensional maps of a microtubule and a kinesin head (3-340 a.a.)-microtubule complex. Surface representation of the computed three-dimensional maps of a microtubule (a), and a kinesin head (3-340 a.a.)-microtubules complex. (From Ref. 12)

Fig. 6. Schematic representation of KIFs and their cargoes in the nerve axon. Anterograde transport: mitochondria are transported by monomeric KIF1B (pink). Synaptic vesicle precursors are transported by monomeric KIF1A (red). KIF3A-KIF3B heterodimer (orange) and kinesin homodimer (green) are conveying unknown membranous organelles distinct from synaptic vesicle precursors. Retrograde transport: multivesicular bodies, prelysosomal membranous organelles and endosomes are transported by brain dynein (purple) and a putative unknown retrograde motor (brown). Retrograde motors such as brain dyneins are conveyed to the nerve terminal by associating with membranous organelles moving in the anterograde direction. The plus-end (fast-growing end) of the microtubule array in the axon points towards the synapse. 


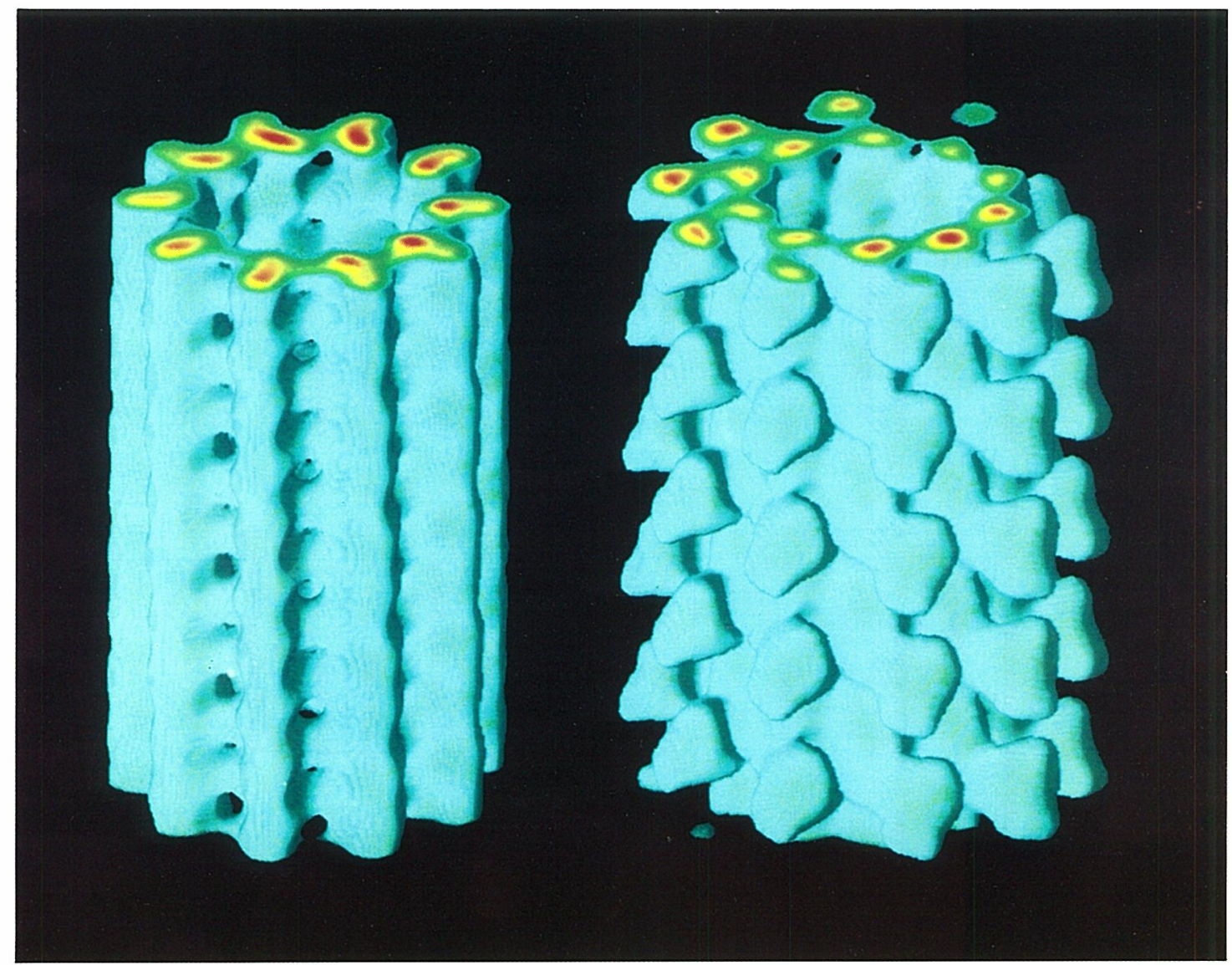

Fig. 5.

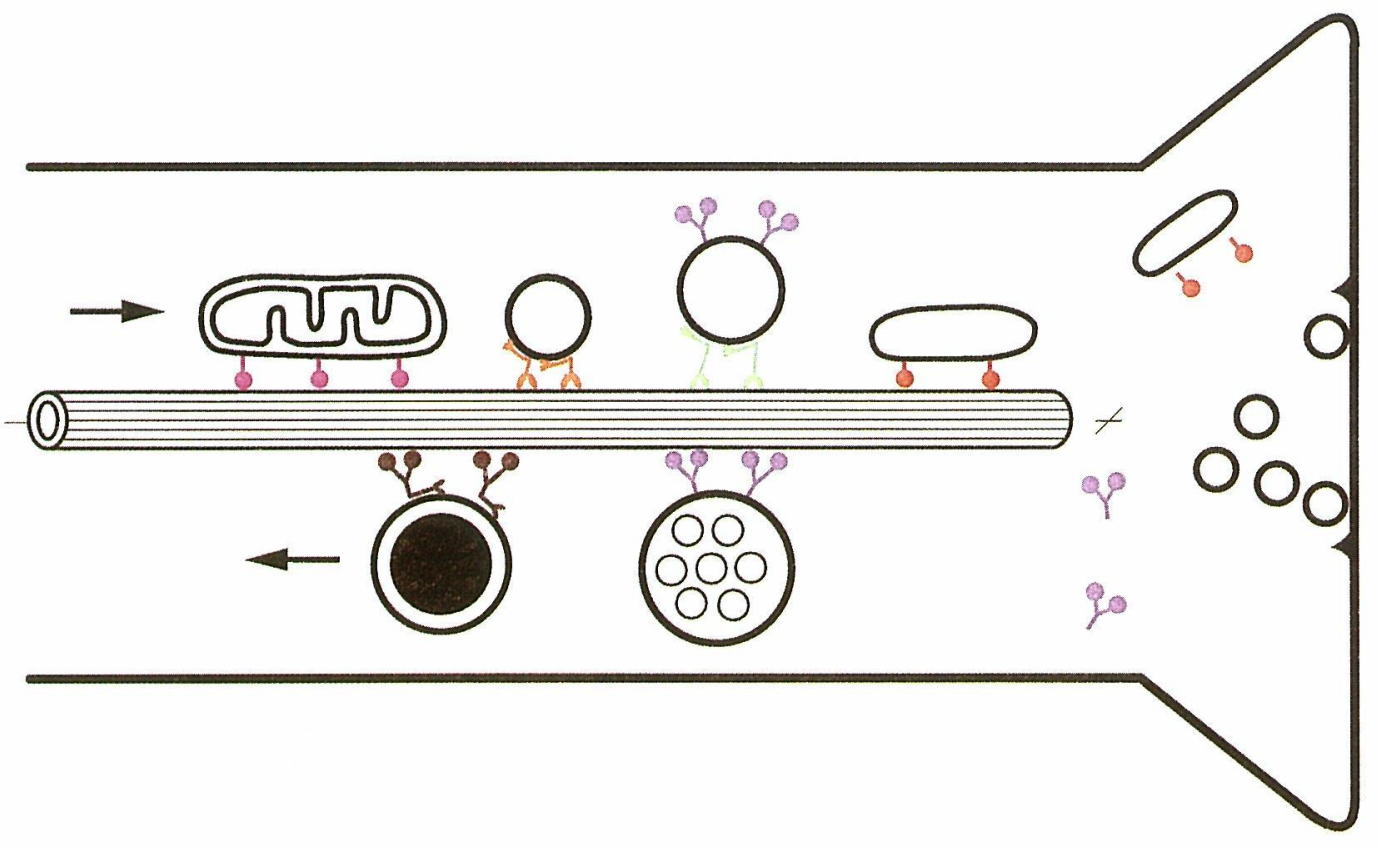

Fig. 6. 
Table I. KIFs RELATED to ORGANELLE TRANSPORTS IDENTIFIED AND CHARACTERIZED IN MURINE TISSUES

\begin{tabular}{|c|c|c|c|c|c|c|c|}
\hline Name & $\begin{array}{c}\text { Molecular } \\
\text { mass }\end{array}$ & $\begin{array}{l}\text { Number of } \\
\text { amino acid }\end{array}$ & $\begin{array}{c}\text { Subunit } \\
\text { structure }\end{array}$ & $\begin{array}{c}\text { Direction of } \\
\text { transport velocity }\end{array}$ & Expression & $\begin{array}{c}\text { Organell } \\
\text { transported }\end{array}$ & Reference \\
\hline KIF1A & $192 \mathrm{kDa}$ & 1695 & $\begin{array}{l}\text { Monomer } \\
\text { N-terminal motor domain } \\
\text { type }\end{array}$ & $\begin{array}{l}\text { Microtubule plus end } \\
1.5 \mu \mathrm{m} / \mathrm{sec}\end{array}$ & Neuron specific & $\begin{array}{l}\text { Subtype of precurosor of } \\
\text { synaptic vesicles }\end{array}$ & 17 \\
\hline KIF1B & $130 \mathrm{kDa}$ & 1150 & $\begin{array}{l}\text { Monomer } \\
\text { N-terminal motor domain } \\
\text { type }\end{array}$ & $\begin{array}{l}\text { Microtubule plus end } \\
0.66 \mu \mathrm{m} / \mathrm{sec}\end{array}$ & Ubiquitous & Mitochondria & 19 \\
\hline KIF2 & $81 \mathrm{kDa}$ & 716 & $\begin{array}{l}\text { Homodimer } \\
\text { central motor domain } \\
\text { type }\end{array}$ & $\begin{array}{l}\text { Microtubule plus end } \\
0.47 \mu \mathrm{m} / \mathrm{sec}\end{array}$ & $\begin{array}{l}\text { Ubiquitous } \\
\text { Expressed in juvenile neuron } \\
\text { but disappear in adult }\end{array}$ & $\begin{array}{l}\text { Vesicles distinct from } \\
\text { synaptic vesicle } \\
\text { precursors }\end{array}$ & 16,21 \\
\hline KIF3A & $80 \mathrm{kDa}$ & 701 & $\begin{array}{l}\text { Heterodimar with KIF3B } \\
\text { Associated Protein KAP3 } \\
\text { N-terminal motor domain }\end{array}$ & Microtubule plus end & $\begin{array}{l}\text { Abundant in neurons, but } \\
\text { expressed } \\
\text { ubiquitously }\end{array}$ & $\begin{array}{l}\text { Vesicles distinct }(90 \sim 160 \\
\mathrm{nm}) \text { from synaptic } \\
\text { vesicle precursors }\end{array}$ & $16,18,34$ \\
\hline KIF3B & $85 \mathrm{kDa}$ & 747 & $\begin{array}{l}\text { Heterodimar with KIF3A } \\
\text { Associated Protein KAP3 } \\
\text { N-terminal motor domain }\end{array}$ & $\begin{array}{l}\text { Microtubule plus end } \\
0.3 \mu \mathrm{m} / \mathrm{sec}\end{array}$ & $\begin{array}{l}\text { Abundant in neurons, but } \\
\text { expressed } \\
\text { ubiquitously }\end{array}$ & $\begin{array}{l}\text { Vesicles distinct }(90 \sim 160 \\
\mathrm{nm}) \text { from synaptic } \\
\text { vesicle precursors }\end{array}$ & 20,34 \\
\hline KIF4 & $140 \mathrm{kDa}$ & 1231 & $\begin{array}{l}\text { Homodimer } \\
\text { N-terminal motor domain } \\
\text { type }\end{array}$ & $\begin{array}{l}\text { Microtubule plus end } \\
0.2 \mu \mathrm{m} / \mathrm{sec}\end{array}$ & $\begin{array}{l}\text { Ubiquitous } \\
\text { Expressed in juvenile neuron } \\
\text { but disappear in adult }\end{array}$ & Vesicles & 22 \\
\hline
\end{tabular}


new KIFs suggests that each kind of organelle may have a separate motor, although there may well be some redundancy (Fig. 6). At the same time, it is clear that there are probably more motors to be discovered as none discovered so far appears to transport precursors of synaptic vesicles containing SV2 or to transport synaptic membranes containing SNAP25 and syntaxin 1A.

\section{Monomeric Motors KIF1A, KIF1B Shed New Light on Mechanism of Motor Movement on Microtubules}

Identification and characterization of KIFs showed that not only are there numerous members of the kinesin superfamily, but that these motors can also occur in different configurations, namely monomeric motors (KIF1A, KIF1B), homodimeric motors (kinesin, KIF2, KIF4, KIF5) and heterodimeric motors (KIF3A-KIF3B) (Figs. 3, 6). These different configurations may also be important in determining the cargo and rate of organelle transports. The monomeric motors (KIF1A, KIF1B) and the heterodimeric motor (KIF3A-KIF3B) with its associated protein (KAP3) are particularly unique. The differences of the location of motor domain, namely, $\mathrm{N}$ terminal motor domain type, central motor domain type and $\mathrm{C}$ terminal motor domain type, are also interesting and important, as these unique configurations tell us something about microtubule-directed transport.

Preocessivity is a characteristic feature of motility along microtubules generated by KIFs in the axon, compared with other motors such as myosins. Membranous organelles in the axon move along a microtubule for certain distance without dissociation from the microtubule 'rail'. The fact that beads coated with kinesins, or kinesin attached to coverslips, at very dilute concentration, can move on a microtubule suggests strongly that a single kinesin dimer is able to perform processive movement along microtubules without dissociation. In fact, it has been reported that multiple (10-100) ATP molecules may be hydrolysed per diffusional encounter with a microtubule, providing evidence that the activity of kinesin is highly processive, with the motor remaining attached to a microtubule through many cycles of ATP hydrolysis (23-26). A single head of kinesin can bind to and dissociate from a microtubule through a cycle of ATP hydrolysis. This suggests a hand-over-hand mechanism for kinesin procession along a microtubule, where one head of a kinesin homodimer remains bound to a microtubule while the other head dissociates from the microtubule, then moves and rebinds to the microtubule $(27,28)$. A similar mechanism has been suggested for other dimeric motors such as myosins (29). However, because it is clear now that monomeric motors (KIF1A, KIF1B) that exist in the native state can move on microtubules and transport membranous organelles such as mitochondria for a certain distance in vitro, it seems likely that a hand-over-hand mechanism is not essential for processive movement of motor molecules. To perform a processive movement, a single-head monomeric motor such as KIF1A or KIF1B must be kept very close to the microtubule during dissociation and movement of the head. How this occurs is not known, but it could be similar to the gravity working for us when we walk by only one leg. The N-terminal domain in KIF1A and KIF1B, which is next to the N-terminal head domain and is highly homologous in KIF1A and KIF1B, may be responsible for keeping the head close enough to the microtubule surface to prevent diffusion away until the next strong microtubule-binding state is acquired (Fig. 2). Nonetheless, multiple monomeric motors on a membranous organelle or a cover glass can cooperate to achieve processive movement, thus acting as if they were multiheaded motors. Further experiments, such as direct visualization of real processive movement of a single monomeric motor on a microtubule or movement of a microtubule on a cover glass coated with KIF1A or KIF1B at a very dilute concentration, are needed to prove definitively that a monomeric motor is capable of processive movement.

How neurons regulate bidirectional transport in the presence of motors, such as kinesin and brain dynein, that have different directionalities but associate with similar cargoes is another important question. Immunocytochemistry of ligated axons and the subcellular fraction approach revealed that a certain fraction of brain dynein could be transported by associating with membranous organelles that themselves were transported in the anterograde direction by microtubule plus-end-directed motors such as kinesin and KIF3A-KIF3B (30). It is possible that most of these motors dissociate from membranous organelles at the nerve terminals and are then degraded, thus allowing retrograde motors to reassociate with organelles transported in the retrograde direction. There is some evidence for the involvement of phosphorylation of the $\mathrm{KHC}$ in its dissociation from membranous organelles in vitro, and in vivo $(31,32)$.

Since the discovery of KIFs has revealed that certain membranous organelles, such as mitochondria and precursors of synaptic vesicles, have their own motors, an important question is how each individual motor identifies its own cargo. One possibility is that there is a binding protein specific to each motor on the membrane of the organelle. In the case of kinesin, kinectin has been identified as a potential candidate for a receptor on ER (35). Another interesting question arises as to the function of associated proteins such as KAP3 for KIF3A and KIF3B. In addition, why KIF3A-KIF3B needs to be a heterodimer in vivo instead of a homodimer is an interesting question. The cargoes of most of KIFs, including $\mathrm{KHC}$, the functions of each KIF and the cell-biological function of KIF-driven transport have still not been clar- 
ified in depth. Molecular genetic approaches, such as introduction of point mutation in KIF cDNA, analysis of the effect of dominant-negative mutants and analysis of mice engineered to lack certain KIF genes by gene targeting, could all be very informative. In this regard, point mutation of ATP-binding motif of the KHC-generated 'rigor' in kinesin and caused selective blockage of anterograde lysosome membrane transport (14); and gene targeting of KIF1A resulted in severe motor and sensory disturbances, based on considerable structural changes in synaptic structures (Y. Yonekawa et al., unpublished). Future investigation of these new questions will certainly lead to a deeper understanding of the mechanisms of organelle transport and the mechanisms of force production and movement by motor proteins, and are underway in our laboratory.

\section{REFERENCES}

1. Hirokawa, N. 1993. Axonal transport and the cytoskeleton Curr. Opi. Neurobiol., 3: 724-731.

2. Hirokawa, N. 1982. The crosslinker system between neurofilaments, microtubules and membranous organelles in frog axons revealed by quick freeze, freeze fracture, deep etching method. J. Cell Biol., 94: 129-142.

3. Vale, R.D., Reese, T.S., and SheEtz, M.S. 1985. Identification of a novel force-generating protein, Kinesin, involved in microtubule-based motility. Cell, 42: 39-50.

4. BRADY, S. 1985. A novel brain ATPase with properties expected for the fast axonal transport motor. Nature (Lond.), 317: 73-75.

5. VAlLEe, R.B. and ShPetner, H.S. 1990. Motor proteins of cytoplasmic microtubules. Ann. Rev. Biochem., 59: 909-932.

6. Bloom, G.S., Wagner, M.C., Pfister, K.K., and Brady, S.T. 1988. Native structure and physical properties of bovine brain kinesin and identification of the ATP-binding subunit polypeptide. Biochemistry, 27: 3409-3416.

7. Hirokawa, N., Bloom, G.S., and Vallee, R.B. 1985. Cytoskeletal architecture and immunocytochemical localization of microtubule-associated proteins in regions of axons associated with rapid axonal transport: The IDPN-intoxicated axon as a model system. J. Cell Biol., 101: 227-239.

8. Hirokawa, N. and Yorifusi, H. 1986. Cytoskeletal architecture in reactivated crayfish axons, with special referance to crossbridges among microtubules and between microtubules and membrane organelles. Cell Motil. and Cytoskel., 6: 458-468.

9. Hirokawa, N., Pfister, K.K., Yorifuji, H., Wagner, M.C., Brady, S.T., and Bloom, G.S 1989. Submolecular domains of bovine brain kinesin identified by electron microscopy and monoclonal antibody decoration. Cell, 56: 867-878.

10. YANG, J.T., LAYMON, R.A., and Goldstein, L.S.B. 1989. A three-domain structure of kinesin heavy chain revealed by DNA sequence and microtubule binding analyses. Cell, 56: 879-889.

11. Kikkawa, M., Ishikawa, T., Nakata, T., Wakabayashi, T., and Hirokawa, N. 1994. Direct visualization of the microtubule lattice seam both in vitro and in vivo. J. Cell Biol., 127: 1965-1971.

12. KikKaWa, M., Ishikawa, T., WaKabayashi, T., and HiRoKaWA, N. 1995. Three-dimensional structure of the kinesin head-microtubule complex. Nature, 376: 274-276.
13. Hirokawa, N., Sato-Yoshitake, R., Kobayashi, N., Pfister, K.K., BloOM, G.S., and Brady, S.T. 1991. Kinesin associates with anterogradely transported membranous organelles in vivo. J. Cell Biol., 114: 295-302.

14. Nakata, T. and Hirokawa, N. 1995. Point mutation of Adenosine triphosphate-binding motif generated rigor kinesin that selectively blocks anterograde lysosome membrane transport. $J$. Cell Biol., 131: 1039-1053.

15. Hirokawa, N. 1993. The neuronal cytoskeleton: its role in neuronal morphogenesis and organelle transport. In Neuronal Cytoskeleton (N. Hirokawa edit.). CRC Press. pp.3-32.

16. Aizawa, H., Sekine, Y., Takemura, R., Zhang, Z., Nangaku, M., and Hirokawa, N. 1992. Kinesin family in murine central nervous system. J. Cell Biol., 119: 1287-1296.

17. Okada, Y., Yamazaki, H., Sekine, Y., and Hirokawa, N. 1995. The neuron-specific kinesin superfamily protein KIF1A is a unique monomeric motor for anterograde axonal transport of synaptic vesicle precursors. Cell, 81: 769-780.

18. Kondo, S., Sato-Yoshitake, R., Noda, Y., Aizawa, H., NaKata, T., MatsuUra, Y., and Hirokawa, N. 1994. KIF3A is a new microtubules-based anterograde motor in the nerve axon. J. Cell Biol., 125: 1095-1107.

19. Nangaku, M., Sato-Yoshitake, R., Okada, Y., Noda, Y., Takemura, R., Yamazaki, H., and Hirokawa, N. 1994. KIF1B; A novel microtubule plus end-directed monomeric motor protein for transport of mitochonndria. Cell, 39: 12091220.

20. Yamazaki, H., NaKata, T., OKada, Y., and Hirokawa, N. 1995. KIF3A/B: A heterodimeric kinesin superfamily protein that works as a microtubule plus end-directed motor for membrane organelle transport. J. Cell Biol., 130: 1387-1399.

21. Noda, Y., Sato-Yoshitake, R., Kondo, S., Nangaku, M., and HIRoKAWA, N. 1995. KIF2 is a new microtubule-based anterograde motor that transports membranous organelles distinct from those carried by kinesin heavy chain or KIF3A/B. J. Cell Biol., 129: 157-167.

22. Sekine, Y., Okada, Y., Kondo, S., Aizawa, H., Takemura, R., and Hirokawa, N. 1994. A novel microtubule-based motor protein (KIF4) for organelle transports, whose expression is regulated developmentally. J. Cell Biol., 127: 187-202.

23. Howard, J., Hudspeth, A.J., and Vale, R.D. 1989. Movement of microtubules by single kinesin molecules. Nature, 342: 154-158.

24. Gilbert, S.P., Webb, M.R., Brune, M., and Johnson, K.A. 1995. Pathway of processive ATP hydrolysis by kinesin. $\mathrm{Na}$ ture, 373: 671-676.

25. HACKNEY, D.D. 1995. Highly processive microtubule-stimulated ATP hydrolysis by dimeric kinesin head domains. Nature, 377: 448-450.

26. Berliner, E., Young, E.C., Anderson, K., Mahtani, H., and GeLLS, J. 1995. Failure of a single-headed kinesin to track parallel to microtubule protofilaments. Nature, 373: 718-721.

27. Schnapp, B.J., Crise, B., Sheetz, M.P., Reese, T.S., and KaHN, S. 1990. Delayed start-up of kinesin-driven microtubule gliding following inhibition by adenosine 5 -[beta, gamma imido] triphosphate. Proc. Natl. Acad. Sci. USA, 87: 1005310057.

28. Block, S.M., Goldstein, L.S., and SchnapP, B.J. 1990. Bead movement by single kinesin molecules studied with optical tweezers. Nature, 348: 348-352.

29. Molley, J.E., Beruns, J.E., Kendrick-Jones, J., Tregear, R.T., and White, D.C.S. 1995. Movement and force produced by a single myosin head. Nature, 378: 209-212. 
30. Hirokawa, N., Sato-Yoshitake, R., Yoshida, T., and KaWAShima, T. 1990. Brain dynein (MAP 1C) localizes on both anterogradely and retrogradely transported membranous organelles. J. Cell Biol., 111: 1027-1037.

31. Sato-Yoshitake, R., Yorifuji, H., InAGaki, M., and Hirokawa, N. 1992. The phosphorylation of kinesin regulates its binding to synaptic vesicles. J. Biol. Chem., 267: 2393023936.

32. Okada, Y., Sato-Yoshitake, R., and Hirokawa, N. 1995. The activation of protein kinase A pathway selectively inhibits anterograde axonal transport of vesicles but not mitochondria transport or retrograde transport in vivo. J. Neurosci., 15: 3053-3064.
33. Hirokawa, N. 1996. Organelle transport along microtubulesthe role of KIFs. Trends Cell Biol., 6: 135-141.

34. Yamazaki, H., Nakata, T., OKada, Y., and Hirokawa, N. 1996. Cloning and characterization of KAP3: a novel kinesin superfamily associated protein of KIF3A/3B. Proc. Natl. Acad. Sci. USA, 93: 8443-8448.

35. Toyoshima, I., Yu, H., Steuer, E.R., and Sheetz, M.P. 1992. Kinectin, a major kinesin-binding protein on ER. $J$. Cell Biol., 118: 1121-1131.

36. Gelles, J., Schnapp, B., and Sheetz, M.P. 1988. Tracking kinesin - driven movements with nanometer-scale precision. Nature, 331: 450-453. 